\title{
Testes Descritivos
}

Saionara Sartor, Clara Mariana Gonçalves Lima, Luiza Zazini Benedito, Daniela Caetano, Flávia Michelon Dalla Nora, Silvani Verruck

https://doi.org/10.4322/mp.978-65-994457-0-5.c4

\section{Resumo}

Metodologias descritivas convencionais consistem na descrição e quantificação das propriedades sensoriais dos alimentos por meio da avaliação sensorial feita por uma equipe de julgadores treinados, tendo como objetivo principal a descrição mais completa possível dos atributos sensoriais. Em sua maioria, os testes descritivos são conduzidos após o levantamento e a descrição dos atributos sensoriais. Assim os julgadores também quantificam os estímulos percebidos empregando para isso escalas. Essas metodologias envolvem aspectos qualitativos, pois a equipe relata as características específicas que descrevem os produtos a serem estudados e, quantitativos, pois avalia a intensidade na qual os atributos são percebidos. Estes testes são amplamente empregados para a avaliação da vida de prateleira de produtos, comparação entre produtos concorrentes, desenvolvimento de novos produtos e controle de qualidade de produtos já disponíveis no mercado além da avaliação da influência de trocas de matérias-primas e de processos produtivos. Diversos testes compõe essa classe de métodos de análise sensorial, sendo que os mais comumente conduzidos na indústria de alimentos serão abordados neste capítulo.

Palavras-chave: Análise Descritiva Quantitativa, Dominância temporal das Sensações, Check-All-That-Apply - CATA, perfil de textura e sabor, perfil livre, tempo-intensidade.

\section{Introdução}

$\mathrm{Na}$ indústria de alimentos, a análise sensorial é elemento chave para identificar as expectativas dos consumidores [1]. A análise sensorial compreende um conjunto de técnicas para medir precisamente atributos sensoriais de produtos a partir de respostas humanas. Tais técnicas utilizam 
princípios oriundos da ciência de alimentos, fisiologia, psicologia e estatística, fornecendo respostas objetivas para as propriedades de alimentos, conforme percebidas pelos cinco sentidos [2].

Testes sensoriais descritivos tradicionais envolvem a detecção (discriminação) e a descrição qualitativa e quantitativa dos atributos sensoriais dos alimentos, por meio da avaliação de uma equipe de julgadores treinados [3]. Os aspectos qualitativos dizem respeito à descrição do produto avaliado, e incluem os atributos que compõem a aparência, o aroma, o sabor e a textura de um alimento, enquanto que os aspectos quantitativos relacionam à intensidade de cada característica sensorial presente no produto. Nas metodologias descritivas, após o levantamento e a descrição dos atributos sensoriais, os julgadores treinados também quantificam os estímulos percebidos [4]. De acordo com diversos autores, dentre os métodos de análise descritiva estão listados a Análise Descritiva Quantitativa (ADQ), perfil de sabor, perfil de textura, perfil de livre escolha, tempo intensidade, análise descritiva de espectro e a Check-All-That-Apply (CATA) [5, 6].

A ADQ proporciona uma completa descrição de todas as propriedades sensoriais de um produto, representando um dos métodos mais completos e sofisticados para caracterização sensorial de atributos importantes [5]. 0 método da ADQ desenvolvido por Stone et al. [7] é muito utilizado para traçar, de forma mais completa possível, o perfil sensorial quanto aos atributos de aparência, odor, textura e sabor. Para tanto, utiliza-se uma equipe de julgadores que desenvolve descritores e emprega escalas para medida de suas intensidades, caracterizando e descrevendo atributos sensoriais das amostras em estudo [4].

As técnicas descritivas são métodos abrangentes e flexíveis, capazes de fornecer informações detalhadas sobre as propriedades sensoriais de um alimento, constituindo-se como uma das mais importantes ferramentas da avaliação sensorial [2]. A descrição quantitativa dos atributos dos alimentos proporciona informações importantes do perfil sensorial possibilitando a sua utilização no controle de qualidade, na comparação de protótipos de alimentos com o intuito de entender o comportamento do consumidor em relação aos atributos sensoriais e também para o mapeamento sensorial de produtos [8, 9]. Também podem ser utilizadas para acompanhar as mudanças do produto ao 
longo do tempo de armazenamento e os efeitos de embalagem, ou ainda, para investigar os efeitos dos ingredientes ou variáveis de processamento na qualidade sensorial final de um produto [4, 10, 11]. A principal importância da análise descritiva é a sua capacidade de permitir a correlação entre medidas sensoriais e instrumentais e/ou a segmentação dos consumidores de acordo com a sua preferência por meio da correlação da avaliação descritiva com testes afetivos $[10,12,13]$.

Métodos específicos refletem várias filosofias e abordagens sensoriais, no entanto, a Análise Descritiva Quantitativa, que pode combinar diferentes abordagens de todos esses métodos, vem sendo frequentemente empregada durante aplicações práticas para atender a objetivos específicos do projeto. Neste capítulo, serão abordados os testes descritivos comumente empregados na análise sensorial clássica, dando maior destaque à $A D Q$, tendo em vista que este é o teste mais empregado para avaliação descritiva de produtos.

\section{Análise Descritiva Quantitativa (ADQ)}

A ADQ é a técnica sensorial descritiva mais utilizada na área de alimentos, pois permite o levantamento, a descrição e a quantificação dos atributos sensoriais detectáveis no produto. Para tanto, avaliadores com alto grau de treinamento realizam a análise sensorial e uma análise estatística robusta dos dados é conduzida [5].

Esta metodologia envolve três etapas fundamentais: i) o levantamento de atributos e a familiarização dos avaliadores com os produtos; ii) a definição, em consenso com a equipe de avaliadores, dos termos descritores e a fixação das referências que servirão como padrões de intensidade (mínima e máxima) para cada atributo, e iii) a avaliação das amostras utilizando, normalmente, uma escala não estruturada de nove centímetros para quantificação da intensidade dos atributos sensoriais. Os dados gerados são transformados em médias de intensidade, por atributo, e avaliados estatisticamente por análise de variância (ANOVA). Nos casos em que diferenças entre as amostras são observadas, testes de comparação de médias, usualmente o Teste de Tukey, são realizados [2].

Para que os resultados sejam satisfatórios, a equipe é frequentemente monitorada com relação ao seu desempenho (concordância, capacidade 
discriminativa, repetibilidade) e à reprodutibilidade, individual ou do grupo como um todo. Estes testes de desempenho são conduzidos durante os treinamentos, com o objetivo de alcançar resultados mais precisos e, portanto, mais confiáveis e consistentes [12, 14].

Alguns pontos importantes devem ser considerados quando uma ADQ é aplicada, sendo que a seleção adequada do painel de avaliadores, geração de atributos, dentre outras características importantes inerentes ao teste são relevantes para o sucesso da análise sensorial. Neste sentido, serão discutidos no decorrer do texto alguns pontos importantes destas etapas prévia a aplicação de uma ADQ.

\subsection{A seleção de um painel de análise descritiva}

$O$ instrumento analítico para avaliação sensorial de uma $A D Q$ é constituído por um painel treinado. Neste sentido, sua confiabilidade depende da objetividade, da precisão e da reprodutibilidade da avaliação dos provadores. A análise descritiva requer controle rigoroso das possíveis variáveis que podem interferir nos resultados, como o ambiente do teste, a relação das amostras e julgadores, seleção dos atributos a serem avaliados, condições dos julgadores e treinamento $[3,15]$.

São parte da seleção e do treinamento de painéis de avaliação sensorial as seguintes etapas: i) recrutamento (por meio de questionários); ii) préseleção; iii) definição de atributos do produto a ser testado (deve ser consensual); iv) definição da ficha sensorial para o produto; v) treinamento e vi) seleção final. Durante a seleção são avaliadas a concordância entre os membros da equipe, o poder discriminativo (de diferenciar as amostras) e a repetibilidade (capacidade de reproduzir resultados) do provador [2].

Para a formação da equipe, a etapa de recrutamento é onde ocorre o primeiro contato com os julgadores, no qual são obtidas informações gerais, assim como são fornecidas informações em relação a como serão conduzidos os testes [1]. O recrutamento pode ser conduzido utilizando questionários ou entrevistas pessoais, e, desde então, os julgadores devem ser informados sobre os objetivos e duração do teste, procedimentos gerais e vantagens do treinamento [3]. $\mathrm{Na}$ seleção dos julgadores, alguns fatores devem ser considerados, como por exemplo, o estado de saúde, presença de alergias, 
disponibilidade e compromisso com o cronograma do trabalho, motivação, criatividade verbal e concentração para a realização dos testes sensoriais. Ademais, indivíduos fumantes não devem compor equipes, tendo em vista que podem apresentar alterações no paladar e também, uma alimentação inadequada, sem a adoção de hábitos saudáveis pode ser uma característica que pode comprometer a qualidade dos resultados obtidos [4].

São utilizados como formas de recrutamento a aplicação de testes sensoriais discriminativos, nos quais características tais como a acuidade sensorial bem como a capacidade discriminatória dos candidatos são avaliadas. Em cada caso, deve ser utilizada a metodologia mais adequada para o objetivo proposto, a fim de formar uma equipe capacitada para a realização das análises e evitar a perda de tempo. É necessário levar em conta o tipo de informação que se deseja obter e as características do produto a ser avaliado para então, selecionar o teste mais adequado para selecionar julgadores aptos $[12,14]$.

Alguns trabalhos discutem sobre os testes de triagem que podem ser aplicados durante o recrutamento (por exemplo, questionários de frequência alimentar), e como o desempenho do painel pode ser monitorado a partir do uso de testes de concentração, de personalidade e de capacidade de discriminação. Usualmente, os testes empregados nesta etapa são o teste triangular, teste duo-trio, teste de ordenação, teste de gostos básicos, reconhecimento de odores e escalas de categoria [12, 14]. Neste mesmo contexto, a literatura reporta vários trabalhos que discutem a seleção de julgadores $[11,13,16,17]$ e os testes mais empregados para seleção e avaliação do desempenho dos julgadores [8, 9, 12, 13, 15].

Ainda no contexto de recrutamento de um painel treinado para a análise sensorial de alimentos é necessário considerar que as habilidades sensoriais variam entre as pessoas e a maioria delas não conhece suas habilidades em relação aos próprios sentidos. No entanto, nem todas as pessoas são qualificadas para todos os testes, lembrando que a participação dos julgadores na avaliação sensorial de um determinado produto deve ser sempre voluntária [5].

Outro ponto de extrema importância para o sucesso geral de uma avaliação sensorial é o compromisso e a motivação dos membros do painel 
pois, independentemente do desempenho dos prováveis julgadores, se eles não puderem comparecer às sessões de treinamento ou avaliação, eles não terão valor para a análise. Para garantir o comprometimento e motivar os julgadores, entrevistas individuais podem ser usadas. A disponibilidade também pode ser determinada preenchendo um cronograma de horas disponíveis por semana, no entanto, os candidatos quase sempre superestimarão sua disponibilidade. A educação, embora não esteja ligada à capacidade de percepção, pode desempenhar um papel na motivação e habilidade dos julgadores em entender a necessidade de um planejamento experimental cauteloso, para atrasos durante a degustação de amostras, para controle de hábitos alimentares antes do atendimento, e assim por diante [4].

Os julgadores devem apresentar um nível razoável de conhecimento sobre atributos sensoriais da amostra, os quais são avaliados através de questionários durante a seleção. A proposta do treinamento é apresentar aos julgadores as mesmas amostras de referência para que sejam descritos os atributos qualitativos, quantitativos e temporais das amostras, utilizando uma escala de intensidade, com extremos mínimos e máximos [5]. A apresentação destes conceitos que descrevem a amostra pelos julgadores é essencial para estabelecer um quadro comum de referência, cujo propósito é padronizar os descritores para determinado produto e remover conotações subjetivas da metodologia sensorial $[8,9,16,17]$.

\subsection{Geração de atributo descritivo}

A fase de treinamento de julgadores para técnicas de análise sensorial descritiva começa com o desenvolvimento de uma linguagem comum que descreve de forma abrangente e precisa, os atributos do produto [14, 16, 17]. Geralmente, um painel desenvolverá a linguagem sensorial por conta própria, e a contribuição de um líder de painel experiente pode ajudar no processo de aprendizagem. O líder deve ser um moderador imparcial cuja função é facilitar a discussão e entendimento entre julgadores, prover materiais de referência para a equipe e facilitar as definições. Um idioma existente na literatura também pode ser adotado, embora se for desenvolvido por outro laboratório, ou em um país ou região diferente, podem ocorrer dificuldades na compreensão e interpretação dos termos [4]. 
Em muitos estudos, o uso de padrões de referência para descrever os atributos sensoriais podem melhorar essas definições, garantindo 0 alinhamento de conceitos em painéis sensoriais [8, 11, 13, 16, 17]. Os padrões de referência podem ser constituídos por qualquer produto químico, ingrediente, especiaria ou produto que demonstre e reflita em estímulos sensoriais específicos para os atributos de aparência, aroma e sensação na boca. A exemplo de padrões de referência, pode ser citado o uso da manteiga com o objetivo de associar com o aroma de gordura láctica, o pão torrado associado ao aroma de fermento e levedura, grama cortada ou pimentão verde para descrever o aroma "verde" ou gramíneo (herbáceo), solução aquosa de cafeína para descrever o gosto amargo, solução de ácido cítrico ou suco de limão para descrever a sensação ácida, uma solução de sacarose para o gosto doce, e assim por diante.

Os atributos gerados deverão ser discutidos em reuniões entre os julgadores e o líder com o objetivo de desenvolver uma linguagem descritiva consensual, além de, sugerir definições para os termos e propor materiais de referências de qualidade e intensidade. Porém, este método pode estar sujeito a distorções da dinâmica de grupo e, em muitos casos, os membros do painel podem não concordar sobre quais atributos selecionar. Neste caso, o líder do painel pode influenciar o processo de seleção do descritor, encorajando ou enfatizando certos atributos que foram relatados na literatura $[4,15]$. $A$ linguagem descritiva final deve ser definida com precisão e coerência, a qual deve conter termos pontuais suficientes para incluir todos os atributos prováveis de serem encontrados na amostra a ser avaliada, porém não deve conter uma quantidade numerosa, de modo a tornar o seu uso confuso.

\subsection{Formação de conceito e treinamento}

Considerada uma etapa de extrema importância, o treinamento tem como objetivo principal aperfeiçoar as habilidades naturais dos julgadores em reconhecer e descrever os atributos e, ao mesmo tempo, familiarizá-los com as técnicas sensoriais de degustação [3]. Uma vez que os termos descritivos são gerados e selecionados, o painel é treinado para usar um "quadro de referência" comum para ilustrar e definir os atributos do produto e sua intensidade. Este quadro possui como característica ser utilizado como ponto 
de referência (quadro de comparação) aos quais os julgadores se referem mentalmente ao avaliar os produtos. Para a condução do treinamento, utilizamse os próprios produtos a serem avaliados, além de que alguns materiais de referência podem ser empregados [2]. Esse treinamento é realizado até que a equipe esteja apta a realizar os testes e quando as respostas apresentem-se confiáveis.

O aspecto fundamental de qualquer sequência de treinamento é fornecer um trabalho estruturado para aprendizagem, com base em fatos demonstrativos, e permitir aos julgadores habilidade e confiança para a execução das análises sensoriais. Durante o treinamento, é buscado não somente aumentar a sensibilidade e reprodutibilidade dos julgadores, como também, fazer com que a equipe trabalhe como um bloco homogêneo e preciso. Com bom treinamento, é possível conseguir um notável aumento da precisão, tanto em nível individual como coletivo [5]. Antes do treinamento, os avaliadores usam seu próprio quadro de referência pessoal para avaliar produtos, qualitativamente, usando suas próprias palavras para descrever as percepções, e quantitativamente, usando suas experiências anteriores para avaliar intensidades. Através do processo de treinamento, os avaliadores adquirem um quadro de referência qualitativo e quantitativo comum, permitindo o uso de uma linguagem padrão para descrever os conceitos sensoriais e, se exigido pelo método, uma escala comum. Deve ser reforçado aos julgadores que eles estão avaliando os produtos no contexto de todos aqueles aos quais eles foram expostos durante as sessões de geração de termos e formação de conceitos [4].

Considerando que os julgadores tenham respostas diferentes ao mesmo estímulo, as diferenças entre as respostas dadas por duas pessoas podem ser causadas por diferença na intensidade das sensações que receberam. Isso se deve ao fato de que seus órgãos sensoriais diferem em termos de sensibilidade, ou ainda em virtude da diferença na interpretação da sensação, devido à falta de conhecimento de determinado sabor, odor ou gosto. Outro ponto que pode causar uma variação de respostas entre os julgadores é a falta de treinamento para expressar a intensidade em escala numérica. Neste sentido, com o uso de uma terminologia adequada e de padrões de referência, acompanhadas de sessão de treinamento, é possível conferir uniformidade na 
interpretação das sensações, desta forma, as respostas poderão ser similares para um mesmo estímulo. Dentre os vários objetivos do treinamento, vale destacar que quando os provadores são treinados, busca-se um maior aprimoramento das suas habilidades em verbalizar as sensações, trabalhar em grupo, e demonstrar reprodutibilidade [2].

Em geral, a condução de procedimentos de treinamento para facilitar 0 alinhamento de conceitos na análise descritiva devem ser realizados de forma mais criteriosa possível. Os procedimentos adotados durante o treinamento irão depender em grande parte do procedimento experimental do método escolhido, do tempo disponível e dos produtos em teste [4].

Neste sentido, Oliver et al. [13] usaram com sucesso uma escala de adequação para permitir que os membros do painel sensorial selecionassem padrões de referência para um vocabulário descritivo de sabor de morangos. Os participantes do painel estavam familiarizados com uma grande variedade de morangos disponíveis no mercado e, posteriormente, concordaram com uma linguagem para descrever a categoria do produto e a variação sensorial. 0 treinamento do painel para desenvolver o léxico descritivo do morango durou seis sessões. O painel então chegou a um consenso sobre a lista final de atributos e, posteriormente, definiu cada um dos termos e determinadas referências de produto para cada um. A lista de atributos final foi reduzida para refletir apenas os atributos principais de sabor e sabor residual do alimento avaliado.

Estudos de Moussaoui e Varela [12], utilizando técnicas de criação de perfis de produtos de consumo e sua ligação com uma Análise Descritiva Quantitativa, relataram um desempenho adequado de um painel que foi treinado por meio de termos descritivos para bebidas quentes. A avaliação descritiva decorreu em 3 sessões, iniciando com a etapa de treinamento, onde uma lista de 42 atributos correspondentes à categoria de bebida quente foi usada para a avaliação. Nesta etapa, os julgadores avaliaram as intensidades percebidas de um par de amostras através de escalas não estruturadas, com intuito de informar se o atributo era percebido pelos julgadores permitindo avaliar o grau de consenso nas avaliações. Caso fossem detectadas discrepâncias nos atributos ou avaliações, era iniciada uma discussão aberta para se chegar a um consenso. Foram selecionados 23 atributos no total. As 
próximas sessões corresponderam a avaliação sensorial e repetição. Para tanto, as amostras foram apresentadas aos julgadores de forma monádica e sequencial, sendo que cada julgador inseriu sua classificação de intensidade registrando em uma escala não estruturada. Por fim, a última sessão foi uma repetição da sessão anterior para verificar o desempenho do painel, bem como a reprodutibilidade e as interações de atributos usadas pelos julgadores.

Neste mesmo contexto, $\mathrm{Ng}$ et al. [9] utilizaram uma $\mathrm{ADQ}$ para descrever o perfil de sabor e de aroma de um doce de abóbora comercial adicionado de suco de groselha preta. O painel já havia sido treinado para avaliar doce de abóboras de groselha usando este mesmo método. No entanto, para garantir a confiabilidade e precisão dos dados, o painel participou de mais seis sessões de treinamento de duas horas para gerar os termos descritivos de atributos de aroma, sabor, aroma e gosto residual, e para verificar o uso de escalas de atributos para a gama de produtos a ser testada. O estágio de geração de atributos identificou 24 atributos que foram reduzidos, por meio de discussão, a uma lista de 15. Dentre os termos utilizados para discriminar os produtos estão presentes os de atributos de sabor e aroma de groselha fresca, groselha preta processada, groselha preta de confeitaria, entre outros, e os gostos ácido, amargo, adstringente, doce natural e doce artificial. Todos os atributos foram avaliados em escala linear não estruturada, ancoradas nas extremidades com "nada intenso" e "muito intenso".

O perfil de sabor e textura de alimentos ácidos como limonada, maçã, queijo de cabra, iogurte natural sem gordura e conserva de pepino foram estudados por Choi e Garza [16] utilizando a ADQ. Os julgadores selecionados foram treinados em 12 sessões, que foram realizadas 4 dias por semana durante 3 semanas. Oito descritores foram selecionados após consenso com os julgadores (doçura, acidez, salgado, amargor, off-flavor, revestimento na boca, cremosidade, crocância), e seus padrões de referência foram determinados.

Para prever o perfil sensorial e a caracterização vinhos espumantes elaborados pelo método tradicional, Sartor et al. [17], utilizaram a ADQ para gerar os termos sensoriais individualmente e descrever os atributos sensoriais de percepção de aparência, aroma e sabor dos vinhos espumantes. O vocabulário sensorial foi construído em duas sessões de avaliação, realizadas 
por especialistas em vinhos, e os descritores foram selecionados por consenso. A partir da lista de atributos sensoriais gerada, os julgadores foram treinados em dias consecutivos com soluções padrão de diferentes concentrações. Em seguida, os julgadores foram orientados a avaliar cada amostra. Panceri et al. [11] também utilizou a ADQ para a seleção de atributos descritivos que caracterizam vinhos tintos elaborados com uvas desidratadas. Os seguintes descritores foram selecionados por consenso: vermelho-rubi e vermelho-laranja como atributos de aparência, e amargor, acidez, doçura, viscosidade, adstringência e sensação alcoólica como atributos de paladar.

\subsection{Escalas para a avaliação sensorial das amostras}

$\mathrm{Na}$ avaliação dos atributos sensoriais dos alimentos são utilizadas escalas, as quais permitem a determinação da intensidade de cada atributo sensorial da amostra. Os métodos descritivos utilizam escalas de intervalo ou de proporção. Essas escalas relacionam uma grandeza com intensidade da sensação, e a direção das diferenças entre as amostras [2]. A partir do uso de escalas é possível descobrir o quanto as amostras diferem entre si, e qual a amostra que apresenta maior intensidade do atributo sensorial que está sendo avaliado. Em contrapartida, a utilização de escalas exige maior treinamento e habilidade dos julgadores [5].

Para a ADQ são utilizadas escalas não estruturadas de nove centímetros de comprimento, ancoradas nos dois extremos com termos que indicam a intensidade do atributo que está sendo avaliado. $\mathrm{Na}$ escala não estruturada a intensidade do atributo avaliado pode ser cuidadosamente marcado em qualquer ponto da escala e também, pelo fato da ausência de valores numéricos, evita erro psicológico do provador, o qual não é induzido a usar números de sua preferência [2]. Uma consideração importante para a $A D Q$ é que não se deve solicitar às equipes treinadas em análise descritiva a avaliação de sabor ou de outro atributo em termos de gostar ou desgostar ou de aceitabilidade.

Após o treinamento e a seleção final dos provadores os testes finais podem ser conduzidos em condições que garantam a individualidade dos provadores, bem como os demais requisitos necessários à avaliação [2]. As amostras são analisadas por cada julgador da equipe, em repetições, utilizando 
a ficha de avaliação desenvolvida em condições que garantam a individualidade e os demais requisitos envolvidos na análise sensorial como, por exemplo, local adequado, balanceamento na apresentação das amostras, dentre outros. Quando o número de amostras é pequeno, as amostras são apresentadas aos julgadores em uma mesma seção, utilizando o delineamento em blocos completos. Caso não seja possível apresentar todas as amostras em uma mesma seção, o delineamento em blocos incompletos é utilizado [5].

\subsection{Análise dos resultados}

A análise dos resultados é uma das etapas mais importantes em uma análise sensorial, tendo em vista que cada método de análise possui suas peculiaridades de avaliação de dados e, para a obtenção de dados confiáveis e adequados é imprescindível que esta etapa seja conduzida de forma correta. Desta forma, inicia-se com a separação das fichas de resposta preenchidas pelos julgadores, estas devem ser organizadas e separadas por julgador. A obtenção dos escores é feita medindo-se a distância que vai desde a extremidade esquerda até a marca feita pelo julgador, na escala não estruturada de nove centímetros. Os resultados são tabulados em forma de escores para cada característica sensorial avaliada, para cada tratamento, em um quadro de dupla entrada de julgadores versus tratamentos [2].

Os resultados obtidos são analisados estatisticamente por meio de análise de variância (ANOVA), e um teste de comparação de médias quando a ANOVA indicar diferença estatística pelo menos entre duas amostras. Além disso, a análise estatística multivariada (MANOVA) e análise das componentes principais (ACP) pode ser aplicada para avaliar a variabilidade dos dados [5, 9, 15, 18]. As fontes de variação mínimas a serem consideradas são amostra, julgadores e interação entre amostra versus provador.

A apresentação dos resultados geralmente é feita em forma de gráfico aranha, onde é plotada a intensidade média de cada atributo, fixando como ponto central o zero. Esse tipo de representação torna possível verificar as similaridades e diferenças de diversos produtos indicados em um mesmo gráfico. Cada atributo de qualidade sensorial do produto é representado em uma linha, com o valor zero da escala situado no centro da figura. A média de cada atributo é marcada no eixo correspondente a cada produto e o perfil 
sensorial é traçado pela conexão dos pontos $[5,11,16]$.

\subsection{Aplicação da Análise Descritiva Quantitativa}

A análise Descritiva Quantitativa (ADQ) é a metodologia sensorial mais utilizada na área de alimentos, pois permite o levantamento, a descrição e a quantificação dos atributos sensoriais no produto, utilizando julgadores com alto grau de treinamento e análise estatística dos dados [5]. Diversos estudos demonstram que esta metodologia possui inúmeras aplicações, como por exemplo, o acompanhamento de produtos concorrentes, testes de armazenamento para verificar possíveis alterações no decorrer do tempo, desenvolvimento de novos produtos, controle da qualidade de alimentos, determinações químicas versus respostas sensoriais, além de ser possível realizar relação entre testes sensoriais e instrumentais. Diversos trabalhos aplicam a ADQ como método de análise sensorial de alimentos, alguns deles serão brevemente descritos abaixo e, estudos recentes empregando este método serão apresentados no último capítulo deste livro.

Um trabalho conduzido para a avaliação sensorial do mel nos permite distinguir, por exemplo, a origem botânica do mesmo, identificar e quantificar certos defeitos oriundos da fermentação, a presença de impurezas, sabores e odores estranhos. Em particular, a avaliação sensorial é importante na verificação da conformidade dos méis uniflorais, uma vez que pode revelar a presença de componentes botânicos que não são detectados por outros métodos analíticos, mas que podem alterar as características sensoriais típicas, de tal forma que o mel não pode ser comercializado como unifloral [19].

Moussaoui e Varela [12], utilizaram técnicas de criação de perfis de produtos de consumo e sua ligação com uma Análise Descritiva Quantitativa de bebidas quentes, como resultado, os atributos do painel treinado refletiram bem a concepção dos consumidores da categoria de produto em estudo. Em geral, as medições descritivas do painel treinado apresentaram melhor desempenho e não podem ser substituídas por técnicas de perfil, particularmente quando atributos complexos estão envolvidos, como a percepção na boca, ou quando pequenas diferenças precisam ser caracterizadas. Resultado semelhante foi encontrado por Richter et al. [8] no estudo do perfil sensorial de pudim de chocolate por meio das duas metodologias (método de ordenação e ADQ), no 
qual observaram maior discriminação na caracterização das amostras quando estas foram avaliadas por julgadores treinados e aplicação de teste de ADQ.

A análise sensorial de um vinho é realizada por "experts" treinados, através de padrões de qualidade desenvolvidos por tradicionais escolas de enologia ou associações de degustadores profissionais. A principal finalidade desta avaliação é o enquadramento da bebida dentro de padrões préestabelecidos. Estes refletem, na maioria dos casos, as características tradicionais de um vinho e compõem sua identidade junto a consumidores das localidades de origem do vinho [11, 18, 17]. Diversos estudos relacionam a composição química com as propriedades sensoriais dos vinhos, de modo a caracterizar o produto, demonstrando a sua qualidade e tipicidade. Estes estudos incluem a caracterização volátil de vinhos utilizando a roda de aromas para descrição sensorial [20], a determinação das características fundamentais de vinhos maturados em barris de carvalho [21], a influência da qualidade e da maturação da uva sobre o produto final $[10,22]$, o efeito da desidratação da uva em condições controladas nas características sensoriais dos vinhos licorosos [11], a caracterização de vinhos espumantes elaborados com novas variedades de uvas brasileiras [17], dentre outras características.

De acordo com estudos de Murray, Delahunty e Baxter [4] a análise descritiva é, sem dúvida, uma das ferramentas mais valiosas no campo da análise sensorial, sendo amplamente utilizada por muitos profissionais da área. No entanto, ainda existem alguns aspectos da metodologia descritiva que precisam ser considerados e discutidos antes de sua aplicação. Como por exemplo, a dificuldade dos julgadores para descrever a intensidade de atributos complexos como o odor e cor. Neste caso, pode ser possível o uso da análise descritiva em conjunto com outros métodos, como a análise sensorial de tempo-intensidade, onde os avaliadores podem ser treinados para pontuar um atributo de cada vez.

A utilização de análises descritivas com crianças e idosos é outro ponto importante que deve ser avaliado dentro de um segmento de mercado específico, pois idosos e crianças possuem diferentes percepções sensoriais. Os atributos de aparência visual da embalagem do produto alimentício são influências poderosas na aceitabilidade, aspectos de forma, cor, design, símbolos, logotipos e nomes de itens também devem ser considerados [4]. 
Por outro lado, as metodologias descritivas convencionais apresentam longo tempo de execução da técnica sensorial, uma vez que requerem treinamento extensivo dos julgadores para se certificar de que o vocabulário e as escalas de avaliação são utilizados de forma consistente e que o painel apresenta consenso, habilidade de discriminar as amostras e repetibilidade de resultados. A fase de treinamento é extensiva e o tempo demandado apresenta-se como um limitante para sua aplicação no contexto prático da indústria [2, 8, 19]. Alguns estudos demonstram que a utilização de técnicas alternativas têm apresentado uma redução satisfatória no tempo do teste de descrição sensorial $[8,9,12,13]$, porém há limitações na sua aplicabilidade devido à avaliação qualitativa das amostras, ou seja, não é possível identificar a magnitude da diferença entre as amostras e, portanto, esses métodos não são recomendados para análises de estabilidade e controle de qualidade. A $A D Q$ representa um dos métodos mais sofisticados para a caracterização sensorial de importantes atributos de alimentos e bebidas.

\section{Perfil de Textura e Sabor}

Perfil de textura e sabor são métodos caracterizados por descreverem de forma quantitativa e qualitativa as amostras. Têm como objetivo caracterizar as propriedades sensoriais dos alimentos. Estes testes podem ser utilizados no desenvolvimento de novos produtos, no controle de qualidade, nas alterações de ingredientes e/ou formulações e na avaliação de produtos durante a estocagem [2].

A maioria das técnicas descritivas existentes necessitam da utilização de julgadores treinados e empregam uma escala não estruturada para avaliar os produtos. Nos métodos de perfil de textura e sabor os julgadores selecionados devem receber o treinamento sobre a definição de textura e sabor, além de conhecerem o procedimento de avaliação e uso de escalas de referência. Devem ser selecionados pela habilidade de discriminação em atributos de textura e sabor [4].

Uma etapa importante e a elaboração de uma lista de termos descritivos sobre a textura e sabor do produto objeto de estudo sob a orientação do líder da equipe. Por meio de um consenso entre os julgadores, os termos descritivos são levantados e, após o treinamento, é aplicada uma ficha de avaliação 
contendo uma escala não estruturada, normalmente com nove centímetros de comprimento [23].

O teste de perfil de sabor é uma técnica de consenso, e as sessões de desenvolvimento e classificação de vocabulário são realizadas durante as discussões em grupo, com os membros do painel considerando aspectos do sabor geral e os componentes detectáveis do sabor dos alimentos. Enquanto que, o teste de textura visa permitir a descrição da textura desde a primeira mordida até a mastigação completa, além de considerar o aspecto temporal dos atributos [4].

De acordo com Alcantara e Freitas-Sá [24], no perfil de sabor, são avaliados:

- Aroma volátil: impressão de uma forma geral do aroma de forma a avaliar o impacto do mesmo;

- Sabor: impressão de uma forma geral do aroma, avaliando o impacto deste; discriminação dos fatores perceptíveis; intensidade de cada fator e a ordem na qual os fatores são percebidos;

- Sabor residual ou "aftertaste".

$\mathrm{Na}$ análise de perfil de textura, a percepção dos atributos de textura pode ser dividida em cinco fases. Na primeira antes ou sem mastigação, em que todos os atributos geométricos e mecânicos, umidade e gordura podem ser percebidos visualmente ou pelo tato. $\mathrm{Na}$ segunda fase ou primeira mordida, são percebidos na boca os atributos mecânicos e geométricos, além de gordura e umidade. A terceira fase inclui a percepção pelos receptores táteis da boca durante a mastigação e/ou absorção do alimento. Já na fase residual, são percebidas as mudanças que ocorrem durante a mastigação e/ou absorção, como o tipo de fragmentação do produto. Na última fase, tem-se a deglutição, que é conceituada como o ato de engolir e é nesta fase que ocorre a percepção de qualquer resíduo remanescente na boca [25].

Normalmente os testes de perfil de textura são conduzidos em mesa redonda, no qual o painel orientado por um líder traça o perfil de textura do produto avaliado. Poucos julgadores fazem parte destas equipes, sendo que 
equipes constituídas por 8 provadores já é suficiente para conduzir a análise sensorial descritiva [2].

De acordo com Meilgaard, Carr e Civille [6], através do perfil de textura, é possível avaliar os seguintes atributos:

- Dureza: Força requerida para a compressão de uma substância entre os dentes (para sólidos) ou ente a língua e o palato (para semi-sólidos);

- Elasticidade: Grau com o qual um produto volta a sua forma original, depois da compressão dos dentes;

- Coesividade: Grau com o qual uma substância é comprimida entre os dentes antes de romper;

- Fraturabilidade: Força com a qual uma amostra esmigalha, racha ou quebra em pedaços;

- Gomosidade: Energia requerida para desintegrar um alimento semi-sólido ao ponto ideal de deglutição. Densidade que persiste durante a mastigação;

- Mastigabilidade: Tempo (segundos) requerido para mastigar uma amostra, a uma velocidade constante de aplicação de força, para reduzi-la a uma consistência adequada para a deglutição.

- Liberação de umidade: Quantidade de umidade que é liberada da amostra.

Algumas limitações para o teste de perfil de textura e sabor são o tempo necessário para treinamento dos avaliadores, a definição de materiais de referência que possam traduzir as percepções ou sensações, e o limitado escopo do vocabulário, o qual pode não ser suficiente para definir a qualidade sensorial do alimento. Considerando-se o aspecto econômico e o tempo consumido para treinamento da equipe de avaliadores, novos estudos vêm apresentando métodos mais versáteis para atender às necessidades da indústria de alimentos. Como exemplos, pode-se citar: Check-All-That-Apply; Perfil Flash, sorting e mapeamento projetivo [24].

Por fim, normalmente estes testes de perfil de sabor e de textura são testes com poucos tratamentos estatísticos, sendo que a interpretação dos dados é feita através da avaliação de gráficos [2]. 


\section{Perfil Livre}

Um teste que permite que o provador use a quantidade e diversidade de termos da forma que quiser é o perfil livre. Este método de análise sensorial não requer um treinamento do painel de julgadores, tendo em vista que assume que os provadores não diferem na forma de perceber as características sensoriais, mas na forma de descrevê-las. Desta forma, essa técnica permite diminuir ou até eliminar as sessões de treinamento e pode ser realizada com consumidores não frequentes [26]. A exigência com os provadores é que eles sejam objetivos e capazes de usar e compreender escalas de intensidade e desenvolver uma lista de atributos e vocabulários consistentes. Aos provadores é permitido que desenvolvam seu próprio vocabulário sobre as percepções sensoriais e, também, utilizar escalas apropriadas [27].

As amostras são apresentadas à equipe e o provador é solicitado a desenvolver sua própria lista de descritores e, posteriormente, as definições. $O$ levantamento de atributos pode ser realizado através do método Rede - "The Kelly Repertory Grid Method' [28]. Muitas vezes pode ocorrer que alguns termos sejam idênticos para os provadores, mas podem ter significado completamente diferente [29]. Os provadores têm a liberdade de utilizar termos descritivos na quantidade e como desejarem [30]. Vale salientar que o perfil livre pode ser utilizado na descrição de similaridades e diferenças em relação a características sensoriais [31].

Após as sessões de levantamento de termos e discussão entre o líder e os julgadores, são definidas as listas de atributos. Com as listas de cada provador são elaboradas as fichas com as escalas de intensidade e, também deve ser elaborada uma lista de definições dos atributos contendo o que os provadores relataram ao líder. Para a condução deste teste, podem ser utilizadas escalas não estruturadas de $9 \mathrm{~cm}$. No que diz respeito a quantidade de amostras, a literatura reporta um mínimo de 2 amostras para que a técnica seja aplicada e os dados podem ser analisados pela Análise Procrustes Generalizada (GPA) [27].

O perfil livre é um teste muito similar a $A D Q$, diferindo em alguns pontos tais como o não treinamento dos julgadores e a presença de uma lista individual de termos descritivos, enquanto que na ADQ a lista de descritores é 
a mesma. Além disso, enquanto a $A D Q$ avalia os dados estatisticamente por ANOVA, no perfil livre uma análise mais robusta é empregada, o GPA.

\section{Tempo-Intensidade}

O teste tempo-intensidade está ganhando espaço devido ao rápido desenvolvimento das tecnologias e informações para coleta de dados, que antes, era seu principal obstáculo. Este teste mede a intensidade e variações dos atributos dos alimentos ao longo do tempo. Alguns atributos de alimentos requerem um certo tempo para se manifestarem, os quais são caracterizados por apresentarem percepção retardada; ou também a sensação permanece mesmo depois do alimento ser engolido, caracterizando alta persistência [32].

Este método avalia, portanto, a distinção temporal de estímulos sensoriais, com o monitoramento das variações da qualidade do estímulo e de sua intensidade em relação ao aspecto temporal, tendo respostas de como um alimento ou produto se comporta para um indivíduo, desde antes do momento em que é colocado na boca até depois de engolir. Assim, a análise consiste em um método descritivo dinâmico, envolvendo o monitoramento da intensidade sensorial ao longo do tempo diante de um produto ou estímulo sensorial. Como resposta dessa análise, são obtidos parâmetros da taxa do início da estimulação, tempo de extinção e a duração total de todo o processo, que pode variar conforme o produto em estudo [33].

As principais características testadas por esse método são odor, aroma e sabor, sendo que textura também vem sendo avaliada. Tem sido usado para testar uma variedade de produtos em relação a essas características, porém, realizada apenas em um número pequeno de atributos ou com número limitado de produtos, uma vez que neste teste apenas um atributo é avaliado por vez [34].

A análise em si pode ser realizada com uma amostra padrão, onde 0 provador utiliza um questionário em escala ou um programa computacional para expressar a intensidade da propriedade analisada. Em seguida, o mesmo procedimento é realizado para as demais amostras a serem testadas. Para avaliação da persistência do estímulo, o provador volta à marcação em direção ao mínimo na medida em que a propriedade desaparece [35]. 
A avaliação sensorial clássica quantifica a resposta sensorial usando um único ponto de medida, os provadores fazem média do tempo ou integram a sua resposta para decodificarem um valor de intensidade único. Já a avaliação sensorial realizada utilizando-se 0 método de tempo-intensidade provê informações sensoriais temporais sobre a sensação percebida (curva T-I) [36].

Os parâmetros das curvas T-I mais frequentemente utilizados nos estudos de tempo-intensidade são: intensidade máxima (Imax); tempo para atingir a intensidade máxima (TImax); e tempo total de duração do estímulo (Ttot). E com o avanço das tecnologias outros parâmetros foram sendo possíveis serem obtidos, tais como: área sob a curva (Área); área sob a curva antes da intensidade máxima $(A)$; área sob a curva após intensidade máxima (B); tempo de duração da intensidade máxima (Platô), tempo antes do estímulo inicial (tempo de reação) (Tlag), tempo de leitura dos dados (Tfinal), taxa de crescimento (Mads), taxa de decrescimento (Mdes), gosto residual calculado através da razão (A/B). A Figura 1 mostra a representação de uma curva tempo-intensidade de uma sensação única e alguns parâmetros que podem ser extraídos [37].

Figura 1. Curva típica tempo-intensidade com alguns parâmetros representados.

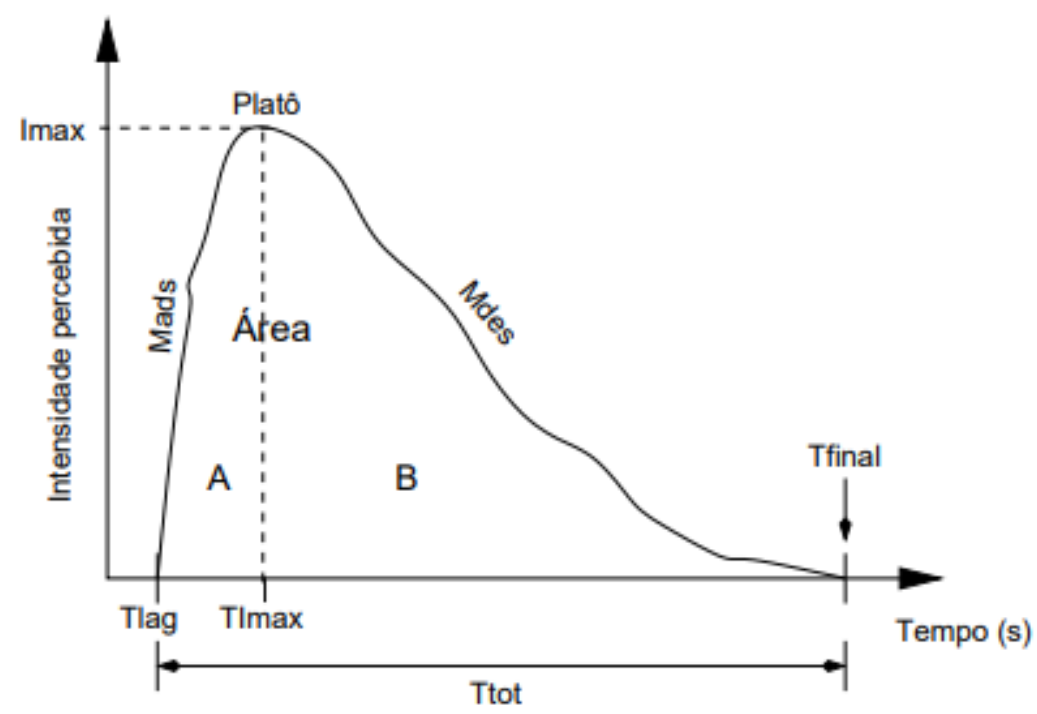

Fonte: Cliff e Heymann [37]. 
Para a análise dos dados de curvas de tempo-intensidade podem ser utilizados diversos métodos, obtendo interpretações e resultados confiáveis e reprodutíveis, sendo os mais utilizados: normalização de dados tempointensidade, proposto por Liu e MacFie [38]; extração de parâmetros ad hoc; análise de componentes principais (ACP); ANOVA com regressão parcial por mínimos quadrados (APLSR); e métodos multivariados alternativos como a ACP duplo e a análise paralela de fatores 2 (PARAFAC2). Todos os métodos possibilitam interpretações dos dados tempo-intensidade e levam a conclusões similares [39].

\section{Análise de Dominância Temporal das Sensações (TDS)}

Como já discutido anteriormente, a análise de tempo-intensidade avalia um único atributo em diferentes amostras, um método dinâmico surgiu a partir deste, porém para analisar diversos atributos. A TDS é um método sensorial descritivo de avaliação continua da "sensação dominante" na qual é considerada como dominante a sensação que chama a atenção do avaliador em determinado tempo, não sendo necessariamente a sensação de maior intensidade. O método TDS considera a multidimensionalidade da percepção ao longo do tempo, possibilitando a avaliação de até 12 atributos simultaneamente considerando suas interações, sendo necessário no mínimo 30 avaliações (provadores $x$ repetições) [40-42].

Neste método é apresentada aos provadores a lista completa de atributos a serem avaliados, utilizando um computador para isso. Em seguida, os provadores recebem as amostras de forma monádica e é solicitado que provem e avaliem os atributos dominantes durante determinado período, podendo alternar esses atributos conforme a percepção. A lista de atributos pode ser gerada por métodos como de Rede utilizado na Análise Descritiva Quantitativa e reduzida por consenso ou análise estatística, sendo recomendado máximo de 10 a 12 atributos, incluindo sabor e textura, com a ordem balanceada entre os avaliadores, sendo mantida na avaliação das amostras de um mesmo avaliador [40-42].

TDS e Temporal Check-All-That-Apply (TCATA) diferem em como as amostras são avaliadas. Enquanto no TDS, os avaliadores devem selecionar apenas o atributo dominante em cada ponto do tempo, no TCATA eles podem 
selecionar todos os atributos que acharem relevantes para descrever a amostra simultaneamente. Estudos anteriores mostraram que, para produtos complexos e com sensações duradouras, ambos os métodos fornecem descrições melhores do que os métodos estáticos, embora difiram no tipo de informação que fornecem [43].

\section{Check-All-That-Apply - CATA}

Entre os vários métodos disponíveis, a análise descritiva e suas variantes tem sido amplamente utilizada quando uma descrição detalhada dos produtos é necessária, são conduzidas com provadores treinados (em sua maioria), com obtenção de resultados satisfatórios. No entanto, as abordagens baseadas no consumidor tornaram-se muito populares na última década, em linha com o reconhecimento da capacidade dos consumidores de caracterizar de forma confiável os produtos [24, 44].

Um dos métodos mais usados para caracterização de produtos sensoriais com os consumidores é o Check-All-That-Apply pela tradução do inglês "marque tudo que se aplique", habitualmente conhecida como CATA. Uma das principais razões para sua popularidade é o fato de que os consumidores consideram a tarefa fácil e não tediosa, uma vez que só são solicitados a selecionar a partir de uma lista pré-definida os atributos que são apropriados para descrever cada amostra focal. Os pesquisadores também consideram as questões do CATA versáteis e simples de implementar [45, 46].

Para a realização do teste CATA são necessárias três etapas principais: levantamento consensual dos descritores; elaboração do questionário CATA; avaliação das amostras pelos consumidores. A pesquisa dos descritores para a lista do questionário CATA pode ser gerada por um painel de avaliadores treinados, por um grupo de foco ou por uma lista anterior de estudos quantitativos do consumidor. Esses descritores não se limitam a atributos sensoriais, podem conter termos relacionados ao uso do produto, conceitos de ajustes e atributos hedônicos [24, 47].

A seleção dos descritores e a quantidade de termos que farão parte do questionário CATA são pontos fundamentais, assim, incluir diferentes termos que se referem às características sensoriais mais relevantes, ao invés de usar muitos termos, a fim de manter a heterogenia do consumidor, é recomentado 
para ter respostas melhores. A quantidade de consumidores para realização da análise é outro fator importante para obter resultados estatisticamente válidos. Desta forma, um número ideal seria entre 50 a 100 consumidores, podendo variar dependendo do tamanho das diferenças entre as amostras, aumentando se essa diferença for pequena [44, 48].

A ordem dos termos apresentados no questionário CATA influencia a resposta do consumidor. Sendo assim, os atributos mais próximos ao topo da lista tendem a ser mais utilizados, sendo recomendado que a ordem seja apresentada de forma balanceada entre os avaliadores e amostras, minimizando a influência dos vieses nas respostas do consumidor, mantendo sua atenção durante toda análise. No entanto, alguns estudos sugeriram que os benefícios do balanceamento entre os avaliadores superam os do balanceamento entre as amostras, pois o consumidor passa a ter familiaridade dos termos e da localização no questionário, assim recomendam a randomização das ordens dos atributos apenas para os avaliadores e não para amostras [45].

Para o CATA utiliza-se um número de amostras entre 1 e 12, dependente da finalidade específica do estudo e das características sensoriais da amostra, levando em consideração a fadiga sensorial. As amostras são apresentadas em sequência monádica, codificadas com números aleatórios de três dígitos, seguindo uma ordem de randomização balanceada para evitar influência da ordem das amostras [44].

A análise dos dados obtidos pelo questionário CATA consistem em dados binários, cuja unidade pode assumir apenas dois estados possíveis, tradicionalmente rotulados como 0 e 1 , indicando se cada consumidor selecionou (1) ou não (0) um determinado termo para descrever cada uma das amostras incluídas no estudo. A relevância de cada termo é determinada pelo cálculo da frequência de seleção, normalmente resumidos em tabelas de contingência que contêm o número do consumidor que selecionou cada termo para descrever cada amostra, e podem ser exibidos em contagens ou porcentagens, sendo o último mais utilizado [48].

A discriminação entre as amostras é verificada aplicando o teste não paramétrico $Q$ de Cochran, verificando a interferência de diferenças de produtos por atributo. Ele avalia, portanto, se os consumidores detectaram 
diferenças significativas entre as amostras para cada um dos termos aplicados no questionário CATA [44, 47, 49].

A análise de correspondência (CA) é amplamente utilizada para tratar dados de uma tabela de contingência, podendo ser considerada uma generalização da análise de componentes principais (PCA) para dados comuns. Esse método projeta os dados em componentes ortogonais para maximizar a representação sequencial da variação nos dados, sendo normalmente apenas dois componentes do gráfico ( $x$ e y) apresentados como forma de mapa. Este mapa, permite visualizar semelhanças e diferenças entre as amostras, bem como suas principais características sensoriais [49].

As questões do CATA, bem como outras abordagens tradicionais de caracterização sensorial, como a análise descritiva, consideram as características sensoriais dos produtos como um fenômeno estático. No entanto, a percepção sensorial é um fenômeno dinâmico à medida que as características sensoriais percebidas dos produtos mudam durante o consumo. Isso pode implicar que, se nenhuma especificação é fornecida aos avaliadores, as características sensoriais dos produtos podem ser avaliadas em diferentes momentos, agregando heterogeneidade nos dados obtidos. Por esta razão, metodologias dinâmicas de caracterização sensorial são necessárias para compreender mais plenamente o curso do tempo das características sensoriais e, consequentemente, das experiências de produtos de consumo $[44,49]$.

Assim, uma extensão das questões do CATA para medir a dinâmica da percepção sensorial, chamada CATA temporal (TCATA), foi introduzida recentemente. Como discutido no anteriormente, no TCATA, os avaliadores recebem uma lista de termos e são solicitados a selecionar continuamente todos os atributos que descrevem as características sensoriais do produto focal durante a avaliação. Eles têm que verificar todas as características sensoriais que percebem em cada momento da avaliação e desmarcar quaisquer atributos selecionados que não sejam mais aplicáveis. Esta metodologia demonstrou uma descrição mais detalhada de como as características sensoriais dos produtos mudam ao longo do tempo $[45,50]$.

O critério exato usado pelos avaliadores em métodos estáticos geralmente não é bem controlado nem capturado com precisão. Por outro lado, os métodos temporais fornecem informações sobre sua avaliação ao longo do 
tempo. Embora ambas as metodologias forneçam informações semelhantes, o TCATA forneceu insights adicionais sobre como as semelhanças e diferenças entre as amostras evoluíram durante o consumo no caso de produtos que experimentam grandes mudanças temporais ou atributos com forte dependência de tempo [51].

Portanto, CATA e TCATA parecem ser metodologias complementares, conforme destacado anteriormente para outras metodologias estáticas e dinâmicas de caracterização sensorial. Não é possível para os consumidores avaliar um grande número de atributos dinamicamente, mas é possível avaliar um grande número de atributos estaticamente. Portanto, pode ser vantajoso fazer com que os consumidores avaliem alguns atributos dinamicamente e outros atributos estaticamente [51, 52].

\section{Considerações finais}

Os testes descritivos são utilizados com o objetivo de descrever as amostras medindo a presença de atributos específicos e da sua intensidade, ou seja, são testes de tanto de caráter qualitativo quanto quantitativo. Os testes sensoriais abordados neste capítulo permitem a realização de uma descrição completa avaliando alterações finas no aspecto sensorial quando por exemplo uma matéria prima ou processo produtivo é alterado. Para isso, diversos testes estão disponíveis, tais como ADQ, CATA, TDS, perfil livre, perfil de textura e de sabor, dentre outros, os quais diferem entre si com relação a diversas características, como por exemplo a exigência de julgadores treinados ou não e até mesmo a forma de análise estatística que é feita. Sendo assim, é de extrema importância o conhecimento das peculiaridades de cada teste para fazer a escolha correta, refletindo na obtenção de dados importantes para que o produto que chega até o consumidor esteja adequado e com as características esperadas por ele.

\section{Referências}

[1] Minim V. P. R. Análise sensorial: estudos com consumidores. 4 ed. Viçosa: Editora UFV; 2018. 332 p.

[2] Dutcosky S. D. Análise sensorial de alimentos. Curitiba: Champagnat; 2019. 
[3] Muñoz A. M., Civille G. V., Carr, B. T. Sensory evaluation in quality control. New York: Van Nostrand Reinhold; 1992.

[4] Murray J., Delahunty C., Baxter I. A. Descriptive sensory analysis: past, present and future. FOOD RESEARCH INTERNATIONAL 2001; 34(6), 461471. https://doi.org/10.1016/s0963-9969(01)00070-9 .

[5] Stone H., Sidel J. L. Sensory evaluation practices. 2 ed. London: Academic Press; 1993. $337 \mathrm{p}$.

[6] Meilgaard M. C., Carr B. T., Civille G. V. Sensory evaluation techniques. CRC press; 2006. 464 p.

[7] Stone H., Sidel J., Oliver S., Woolsey A., Singleton R. C. Sensory evaluation by quantitative descriptive analysis. FOOD TECHNOLOGY 1974; 28:24-34. https://doi.org/10.1002/9780470385036.ch1c.

[8] Richter V. B., De Almeida, T. C. A., Prudencio S. H., De Toledo Benassi M. Proposing a ranking descriptive sensory method. Food Quality and Preference 2010; 21(6):611-620. https://doi.org/10.1016/j.foodqual.2010.03.011.

[9] Ng M., Lawlor J. B., Chandra S., Chaya C., Hewson L. Hort J. Using quantitative descriptive analysis and temporal dominance of sensations analysis as complementary methods for profiling commercial blackcurrant squashes. Food Quality and Preference 2012; 25(2), 121-134. https://doi.org/10.1016/j.foodqual.2012.02.004.

[10] Cadot Y., Caillé S., Samson A., Barbeau G., Cheynier V. Sensory representation of typicality of Cabernet franc wines related to phenolic composition: impact of ripening stage and maceration time. Analytica Chimica Acta 2012; 732:91-99. https://doi.org/10.1016/j.foodqual.2011.08.012.

[11] Panceri C. P., De Gois J. S. Borges D. L. G., Bordignon-Luiz M. T. Effect of grape dehydration under controlled conditions on chemical composition and sensory characteristics of Cabernet Sauvignon and Merlot wines. LWT - Food Science and Technology 2015; 63(1):228-235. https://doi.org/10.1016/j.lwt.2015.02.014.

[12] Moussaoui K. A., Varela P. Exploring consumer product profiling techniques and their linkage to a quantitative descriptive analysis. Food Quality and Preference 2010; 21(8):1088-1099. https://doi.org/10.1016/j.foodqual.2010.09.005. 
[13] Oliver P., Cicerale S., Pang E., Keast R. A Comparison of Temporal Dominance of Sensation (TDS) and Quantitative Descriptive Analysis (QDATM) to Identify Flavors in Strawberries. Journal of Food Science 2018; 83:10941102. https://doi.org/10.1111/1750-3841.14096.

[14] Moskowitz H.R. Applied sensory analysis of foods. Boca Ratton: CRC Press; 1988. 259 p.

[15] Rossini K., Anzanello M. J., Fogliatto F. S. Seleção de atributos em avaliações sensoriais descritivas. Produção 2012; 22(3):380-390. http://dx.doi.org/10.1590/S0103-65132012005000032.

[16] Choi S. E., Garza J. Effects of different miracle fruit products on the sensory characteristics of different types of sour foods by descriptive analysis. Journal of Food Science 2020. https://doi.org/10.1111/1750-3841.14988.

[17] Sartor S., Burin V. M., Caliari V., Bordignon-Luiz M. T. Profiling of free amino acids in sparkling wines during over-lees aging and evaluation of sensory properties. LWT - Food Science and Technology 2021; 140:110847. https://doi.org/10.1016/j.lwt.2020.110847.

[18] Cadot Y., Caillé S., Samson A., Barbeau G., Cheynier V. Sensory dimension of wine typicality related to a terroir by Quantitative Descriptive Analysis, Just About Right analysis and typicality assessmen. Analytica Chimica Acta 2010; 660:53-62. https://doi.org/10.1016/j.aca.2009.10.006.

[19] Mantilla S. P. S., Santos É. B., De Barros L. B., De Freitas M. Q. Análise descritiva quantitativa aplicada em mel de abelhas (Apis mellifera): uma revisão. Colloquium Agrariae 2012; 8(2):75-84. https://doi.org/10.5747/ca.2012.v08.n2.a081.

[20] Capone S., Tufariello M., Siciliano P. Analytical characterisation of Negroamaro red wines by "aroma wheels". Food Chemistry 2013; 141:29062915. https://doi.org/10.1016/j.foodchem.2013.05.105.

[21] Alañón M. E., Schumacher R., Castro-Vázquez L., Díaz-Maroto I. J., DíazMaroto M. C., Pérez-Coelho M. S. Enological potential of chesnut wood for aging Tempranillo wines part I: Volatile compounds and sensorial properties. Food Research International 2013; 51(1):325-334. https://doi.org/10.1016/j.foodres.2012.12.007.

[22] Gonzalo-Diago A., Dizy M., Fernández-Zurbano P. Contribution of low molecular weight phenols to bitter taste and mouthfeel properties in red wines. 
Food

Chemistry

2014

154:187-198.

https://doi.org/10.1016/j.foodchem.2013.12.096.

[23] Poiani M. R., Montanuci F. D. Caracterizações físicas e tecnológicas e perfil de textura de cookies de farinha de uva e linhaça. Brazilian Journal of Food Technology 2019; 22(1):1-14. https://doi.org/10.1590/1981-6723.07418.

[24] Alcantara M., Freitas-Sá D. G. C. Metodologias sensoriais descritivas mais rápidas e versáteis - uma atualidade na ciência sensorial. Brazilian Journal of Food Technology 2018; 21(0). https://doi.org/10.1590/1981-6723.17916.

[25] Associação Brasileira de Normas Técnicas. NBR ISO 11036: Análise 336 sensorial e perfil de textura. Rio de Janeiro; 2017.

[26] Leal S. F. L. S. Análise sensorial do mel usando a técnica de perfil livre e sua aplicação em concursos de mel. Trabalho de Conclusão de Curso (Tecnologia em Alimentos): Universidade Tecnológica Federal do Paraná, Londrina; 2014. 48 p. http://repositorio.roca.utfpr.edu.br/jspui/handle/1/2509.

[27] Verruma-Bernardi M. R., Damásio M. H. Análise descritiva de perfil livre em queijo mozarela de leite de búfala. Ciência e Tecnologia De Alimentos 2004; 24(4):536-542. https://doi.org/10.1590/S0101-20612004000400010.

[28] Moskowitz H. R. Product testing and sensory evaluation of foods: marketing and R\&D approaches. Food \& Nutrition Press, Inc.; 1983. 605 p.

[29] MacFie H. J. H. Assessment of the sensory properties of food. Nutrition Reviews 2009; 48(2):87-93. https://doi.org/10.1111/j.17534887.1990.tb02910.x.

[30] Williams A. A., Langron S. P. The use of free-choice profiling for the evaluation of commercial ports. Journal of The Science of Food and Agriculture 1984; 35(5), 558-568. https://doi.org/10.1002/jsfa.2740350513.

[31] Oliveira A. P. V., Benassi M. T. Avaliação sensorial de pudins de chocolate com açúcar e dietéticos por perfil livre. Ciência e Agrotecnologia 2010; 34(1):146-154. https://doi.org/10.1590/S1413-70542010000100019.

[32] Azevedo B. M., Schmidt F. L., Bolini H. M. A. High-intensity sweeteners in espresso coffee: ideal and equivalent sweetness and time-intensity analysis. International Journal of Food Science \& Technology 2015; 50(6):13741381. https://doi.org/10.1111/ijfs. 12774. 
[33] Lee W. E. III., Pangborn M. Time-intensity: the temporal aspects of sensory perception. Food Technology 1986, 40(11):71-82. https://agris.fao.org/agrissearch/search.do?recordID=US8726877.

[34] Kemp S. E., Hort J., Hollowood T. Descriptive analysis in sensory evaluation. Ed. 1. Willey Blackwell: India; 2018.

[35] Cadena R. S., Bolini H. M. A. Time-intensity analysis and acceptance test for traditional and light vanilla ice cream. Food Research International 2011; 44(3):677-683. https://doi.org/10.1016/j.foodres.2010.12.012.

[36] Chaya C., Perez-Hugalde C., Judez L., Wee C. S., Guinard J.-X. Use of the STATIS method to analyze time-intensity profiling data. Food Quality and Preference 2004; 15(1):3-12. https://doi.org/10.1016/s0950-3293(02)00219-7.

[37] Cliff M., Heymann H. Development and use of time-intensity methodology for sensory evaluation: A review. Food Research International 1993; 26(5):375385. https://doi.org/10.1016/0963-9969(93)90081-s.

[38] Liu Y.-H., MacFie H. J. H. Methods for averaging time-intensity curves. Chemical Senses 1990; 15(4), 471-484. https://doi.org/10.1093/chemse/15.4.471.

[39] Ovejero-López I., Bro R., Bredie W. L. P. Univariate and multivariate modelling of flavour release in chewing gum using time-intensity: a comparison of data analytical methods. Food Quality and Preference 2005; 16(4):327-343. https://doi.org/10.1016/j.foodqual.2004.05.014.

[40] Pineau N., Schlich P., Cordelle, S. Mathonnière C., Issanchou S., Imbert A., Rogeaux M., Etiévant P., Köster E. Temporal Dominance of Sensations: Construction of the TDS curves and comparison with time-intensity. Food Quality and Preference 2009; 20(6):450-455. https://doi.org/10.1016/j.foodqual.2009.04.005.

[41] Rodrigues J. F., Souza V. R. De Lima R. R., Carneiro J. de D. S., Nunes C. A., Pinheiro A. C. M. Temporal dominance of sensations (TDS) panel behavior: A preliminary study with chocolate. Food Quality and Preference 2016; 54:5157. https://doi.org/10.1016/j.foodqual.2016.07.002.

[42] ISO. International Organization for Standardization. Sensory analysis Methodology - General guidance for establishing a sensory profile. ISO standard 13299:2017. Geneva, Switzerland: International Organization for Standardization; 2017. 
[43] Ares G. Jaeger S. R., Antúnez L., Vidal L., Giménez A., Coste B., Picollo A., Castura J. C. Comparison of TCATA and TDS for dynamic sensory characterization of food products. Food Research International 2015; 78:148158. https://doi.org/10.1016/j.foodres.2015.10.023.

[44] Jaeger S. R., Beresford M. K., Paisley A. G., Antúnez L., Vidal, L., Cadena R. S., Giménez A., Ares G. Check-all-that-apply (CATA) questions for sensory product characterization by consumers: Investigations into the number of terms used in CATA questions. Food Quality and Preference 2015; 42:154-164. https://doi.org/10.1016/j.foodqual.2015.02.003.

[45] Tiyo de Godoy N., Veneziano A. L., da Cunha Rodrigues L., Schoffen Enke D. B., Lapa-Guimarães J. QIM, CATA, and Word Association methods for quality assessment of flathead gray mullet (Mugil cephalus): Going beyond the trained panel. Journal of Sensory Studies 2019; 34(2):e12482. https://doi.org/10.1111/joss.12482.

[46] Tiepo C. B. V., Werlang S., Reinehr C. O., Colla L. M. Sensory methodologies used in descriptive studies with consumers: Check-All-ThatApply (CATA) and variations. Research, Society and Development 2020; 9(8):e407985705-e407985705. https://doi.org/10.33448/rsd-v9i8.5705.

[47] Kim I., Lee Y. Recent trends in check-all-that-apply (CATA) method for food industry applications. Food Science and Industry 2019; 52(1):40-51. https://www.koreascience.or.kr/article/JAKO201913747256570.page.

[48] Llobell F., Cariou V., Vigneau E., Labenne A., Qannari E. M. A new approach for the analysis of data and the clustering of subjects in a CATA experiment. Food Quality and Preference 2018; 72:31-39. https://doi.org/10.1016/j.foodqual.2018.09.006.

[49] Alexi N., Nanou E., Lazo O., Guerrero L., Grigorakis K., Byrne D. V. CheckAll-That-Apply (CATA) with semi-trained assessors: Sensory profiles closer to descriptive analysis or consumer elicited data? Food Quality and Preference 2018; 64:11-20. https://doi.org/10.1016/j.foodqual.2017.10.009.

[50] Ares G., Jaeger S. R. Check-all-that-apply (CATA) questions with consumers in practice: Experimental considerations and impact on outcome. Rapid Sensory Profiling Techniques 2015; 0:227-245. https://doi.org/10.1533/9781782422587.2.227. 
[51] Vidal L., Antúnez L., Ares G., Cuffia F., Lee P.-Y., Le Blond M., Jaeger S. $R$. Sensory product characterisations based on check-all-that-apply questions: Further insights on how the static (CATA) and dynamic (TCATA) approaches perform. Food Research International 2019; 125:108510. https://doi.org/10.1016/j.foodres.2019.108510.

[52] Alcaire F., Antúnez L., Vidal L., Zorn S., Giménez A., Castura J. C., Ares G. Comparison of static and dynamic sensory product characterizations based on check-all-that-apply questions with consumers. Food Research International 2017; 97:215-222. https://doi.org/10.1016/j.foodres.2017.04.012.

\section{Autores}

Saionara Sartor ${ }^{1}$, Clara Mariana Gonçalves Lima², Luiza Zazini Benedito², Daniela Caetano ${ }^{3}$, Flávia Michelon Dalla Nora ${ }^{4}$, Silvani Verruck ${ }^{5, *}$

1. Departamento de Ciência e Tecnologia de Alimentos, Universidade Tecnológica Federal do Paraná, 85601-970, Francisco Beltrão, PR, Brasil.

2. Departamento de Ciência de Alimentos, Universidade Federal de Lavras, 37.200-00, Lavras, MG, Brasil.

3. Instituto Federal de Educação Ciência e Tecnologia do Norte de Minas Gerais - Campus Salinas, 39400-149, Minas Gerais, MG, Brasil.

4. Universidade Federal de Santa Maria, Departamento de Tecnologia e Ciência dos Alimentos, 97105-900, Santa Maria, RS, Brasil.

5. Universidade Federal de Santa Catarina, Departamento de Ciência e Tecnologia de Alimentos, 88034-000, Florianópolis, SC, Brasil.

*Autor para correspondência: silvani.verruck@ufsc.br 